\title{
Analysis of the order of accuracy for node-centered finite volume schemes
}

\author{
Sofia Eriksson and Jan Nordström
}

March 10, 2009

\begin{abstract}
The order of accuracy of the node-centered finite volume methods is analyzed, and the analysis is based on an exact derivation of the numerical errors in one dimension. The accuracy for various types of grids are considered. Numerical simulations and analysis are performed for both a hyperbolic and a eliptic case, and the results agree. The impact of weakly imposed boundary conditions is analyzed and verified numerically. We show that the error contribution from the primal and dual grid can be treated separately.
\end{abstract}

\section{Introduction}

The order of accuracy of the widely used $[11,3,8,4,16,9]$ node-centered finite volume method (FVM) has been discussed recently. There are indications that the scheme yields at least first order accuracy for all types of grids [2, 14], while others have indications that for some meshes the order is less than one $[12,15,6]$. In this report the behaviour of the node-centered finite volume method on a one dimensional mesh is analyzed, using both hyperbolic and elliptic model problems. The scheme is formulated in terms of Summation-By-Parts operators and the boundary conditions are imposed weakly using penalty terms $[1,7,10,13]$.

The advantage with the one-dimensional analysis is that we can exactly compute the discretization error in terms of the truncation error. No assumptions or non-sharp estimates are needed. The drawback is of course that most applications are in multiple dimensions. However, without the direct link from truncation error to discretization error, no real understanding is possible. To be crystal clear, one has to be able to invert the operators.

The outline of this paper is as follows. First we make sure that our numerical procedure is stable for time dependent problems. Next, numerical simulations are performed for one hyperbolic and one elliptic ordinary differential equation (ODE), in order to find the rates of convergence. This is done using several types of grids. Thereafter the two problems are thoroughly analyzed, and the orders of accuracy obtained analytically is compared to the rates of convergence obtained experimentally. 


\section{Well-posedness and stability}

Even though we here primarily focus on steady solutions we will first make sure that the corresponding time-dependent problem is well-posed and stable. This procedure guarantees that one can solve for the steady solution and that the matrix in the resulting linear equation system can always be inverted.

\section{$2.1 \quad$ Well-posedness}

The continuous problem that we consider is

$$
\begin{aligned}
& u_{t}+a u_{x}=\varepsilon u_{x x}+F(x, t), \quad 0 \leq x \leq 1 \\
& u(x, 0)=f(x),
\end{aligned}
$$

where $\varepsilon \geq 0, F$ is a forcing function and $f$ is the initial condition. The problem in (1) is strongly well-posed if the solution is bounded in terms of all the data $F, f, g_{0}, g_{1}$, where $g_{0}$ and $g_{1}$ are the boundary data at the left and right boundary, respectively. We limit ourselves to show well-posedness, hence considering the data $F, g_{0}, g_{1}=0$, see [5].

To examine this we use the energy method. We multiply the differential equation in (1) by $2 u$ and integrate over the spatial domain. We obtain

$$
\frac{d}{d t}\|u\|^{2}=a u(0, t)^{2}-a u(1, t)^{2}+2 \varepsilon\left(-u(0, t) u_{x}(0, t)+u(1, t) u_{x}(1, t)-\left\|u_{x}\right\|^{2}\right)
$$

where $\|u\|^{2} \equiv \int_{0}^{1} u^{2} d x$. If $\varepsilon=0$ the differential equation will be hyperbolic and we will only need one boundary condition. If $a>0, u(0, t)=g_{0}$ has to be given, if $a<0$, $u(1, t)=g_{1}$ has to be given instead. Assuming $a>0$ and $g_{0}=0$ we get

$$
\frac{d}{d t}\|u\|^{2}=-a u(1, t)^{2}
$$

Considering the parabolic case we have $\varepsilon \neq 0$ and hence we have to give data at both boundaries, i.e. $u(0, t)=g_{0}$ and $u(1, t)=g_{1}$. Inserting the zero boundary data yields

$$
\frac{d}{d t}\|u\|^{2}=-2 \varepsilon\left\|u_{x}\right\|^{2} .
$$

Time integration of (3) and (4) gives that the problem in (1) is well-posed in the classical sense, assuming that the correct number of boundary conditions is used.

\subsection{The discrete formulation}

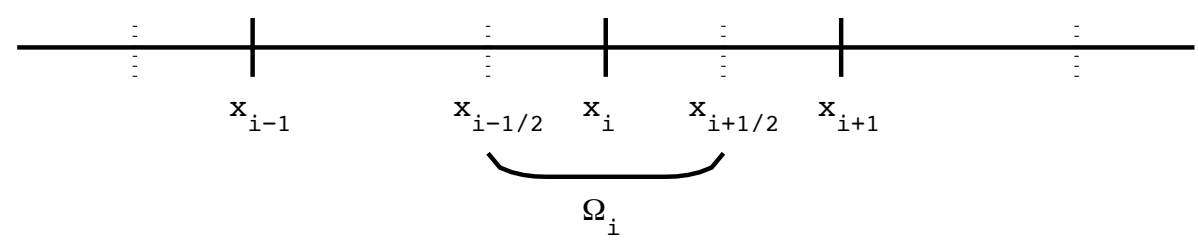

Figure 1: The control volume $\Omega_{i}$ on an unstructured mesh. The unknowns are located at positions with an integer index, i.e. $x_{i-1}, x_{i}, x_{i+1}$. The positions of the flux points are denoted with half indices, e.g. $x_{i-1 / 2}$ and $x_{i+1 / 2}$. The control volumes $\Omega_{i}$ are defined as $x_{i+1 / 2}-x_{i-1 / 2}$. 
To discretize we integrate the differential equation in (1) over the control volumes, $\Omega_{i}$. The volumes $\Omega_{i}$ corresponds to positions $x_{i}$ and are defined as $\Omega_{i}=x_{i+1 / 2}-x_{i-1 / 2}$, where $x_{i+1 / 2}$ is the flux point between the positions $x_{i}$ and $x_{i+1}$, see Figure 1 . At the boundaries we have $\Omega_{0}=\left(x_{1 / 2}-x_{0}\right)$ and $\Omega_{N}=\left(x_{N}-x_{N-1 / 2}\right)$.

Next the term $\int_{\Omega_{i}} u_{t} d x$ is approximated by $\Omega_{i} u_{t}\left(x_{i}\right)$, and the flux on the control volume boundaries by centered approximations. The numerical scheme becomes

$$
\Omega_{i}\left(v_{i}\right)_{t}=-a \frac{\left(v_{i+1}-v_{i-1}\right)}{2}+\varepsilon\left(\frac{v_{i+1}-v_{i}}{x_{i+1}-x_{i}}-\frac{v_{i}-v_{i-1}}{x_{i}-x_{i-1}}\right)+\Omega_{i} F
$$

where $v_{i}$ denotes the discrete representation of $u\left(x_{i}\right)$. In matrix form, including a weak implementation of the boundary conditions, this is written as

$$
\begin{aligned}
v_{t} & =-a P^{-1} Q v+\varepsilon P^{-1}(-A+B S) v+F \\
& +\tau_{0} P^{-1} e_{0}\left(v_{0}-g_{0}\right)+\tau_{N} P^{-1} e_{N}\left(v_{N}-g_{N}\right) \\
v(0) & =f
\end{aligned}
$$

where $v=\left[\begin{array}{llll}v_{0} & v_{1} & \ldots & v_{N}\end{array}\right]^{T}$ is a vector containing the discretized numerical solution. We have also introduced the vectors $e_{0}=\left[\begin{array}{llll}1 & 0 & \ldots & 0\end{array}\right]^{T}$ and $e_{N}=\left[\begin{array}{llll}0 & \ldots & 0 & 1\end{array}\right]^{T} . P^{-1} Q$ represents $d / d x$ and $P^{-1}(-A+B S)$ represents $d^{2} / d x^{2}$. The boundary conditions are included using a weak penalty treatment, see $[1,10,13]$ for details. The penalty parameters are denoted $\tau_{0, N}$. P contains the control volumes $\Omega_{i}$. The difference operator $Q$ is nearly skew-symmetric as $Q+Q^{T}=B$, where $B=E_{N}-E_{0}$. $E_{0}$ and $E_{N}$ are matrices containing only zeros, except for $E_{0}(0,0)=E_{N}(N, N)=1$. $P$ and $Q$ are shown in (7).

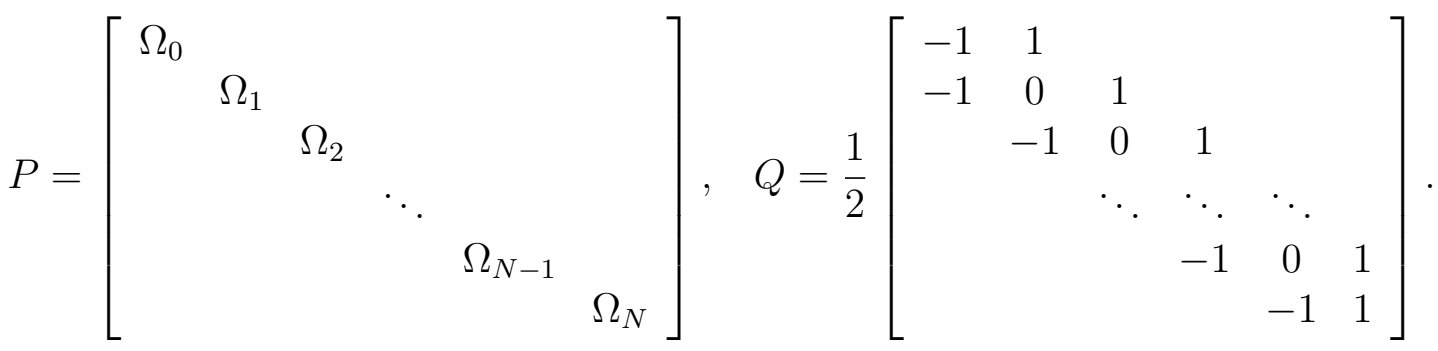

Here $S$ is such that $(S v)_{0}=\left(v_{1}-v_{0}\right) / \Delta x_{1}$ and $(S v)_{N}=\left(v_{N}-v_{N-1}\right) / \Delta x_{N}$. The interior of $S$ is equal to the identity matrix. $A$ and $S$ are given in (8), where $\Delta x_{i}=x_{i}-x_{i-1}$.

$$
A=\left[\begin{array}{ccccc}
\frac{1}{\Delta x_{1}} & \frac{-1}{\Delta x_{1}} & & & \\
\frac{-1}{\Delta x_{1}} & \frac{1}{\Delta x_{1}}+\frac{1}{\Delta x_{2}} & \frac{-1}{\Delta x_{2}} & & \\
& \frac{-1}{\Delta x_{2}} & \frac{1}{\Delta x_{2}}+\frac{1}{\Delta x_{3}} & \frac{-1}{\Delta x_{3}} & \\
& \ddots & \ddots & \ddots & \\
& \frac{-1}{\Delta x_{N-1}} & \frac{1}{\Delta x_{N-1}}+\frac{1}{\Delta x_{N}} & \frac{-1}{\Delta x_{N}} \\
& & \frac{-1}{\Delta x_{N}} & \frac{1}{\Delta x_{N}}
\end{array}\right], S=\left[\begin{array}{ccccc}
\frac{-1}{\Delta x_{1}} & \frac{1}{\Delta x_{1}} & & & \\
& 1 & & & \\
& 1 & & \\
& & \ddots & & \\
& & 1 & \\
& & \frac{-1}{\Delta x_{N}} & \frac{1}{\Delta x_{N}}
\end{array}\right] .
$$

\subsection{Stability in the discrete case}

For simplicity we again let $F=g_{0}=g_{N}=0$. We multiply (6) by $v^{T} P$ from the left, add the transpose, introduce $A=S^{T} R S$ and use the relation $\frac{d}{d t}\left(\|v\|_{P}^{2}\right)=\frac{d}{d t}\left(v^{T} P v\right)$. 
That yields

$$
\begin{aligned}
\frac{d}{d t}\left(\|v\|_{P}^{2}\right) & \left.=a\left(v_{0}^{2}-v_{N}^{2}\right)+2 \varepsilon\left(-v_{0}(S v)_{0}+v_{N}(S v)_{N}\right)-2 \varepsilon(S v)^{T} R(S v)\right) \\
& +2 \tau_{0} v_{0}^{2}+2 \tau_{N} v_{N}^{2}
\end{aligned}
$$

First consider the hyperbolic case. In the continuous version we had $a>0$ and should hence only give data at the left boundary. In the discrete case, having $\varepsilon=0$ and $a>0$, the corresponding action is to let $\tau_{N}=0$. We get

$$
\frac{d}{d t}\left(\|v\|_{P}^{2}\right)=v_{0}^{2}\left(a+2 \tau_{0}\right)-a v_{N}^{2}
$$

In the hyperbolic case the semi-discrete scheme in (6) will be stable if $\tau_{0} \leq-a / 2$. For the parabolic case we will use another technique, see [1]. Rewrite (9) as

$$
\begin{aligned}
\frac{d}{d t}\left(\|v\|_{P}^{2}\right) & =-2 \varepsilon(S v)^{T} \tilde{R}(S v) \\
& +\left[\begin{array}{c}
v_{0} \\
(S v)_{0}
\end{array}\right]^{T}\left[\begin{array}{cc}
a+2 \tau_{0} & -\varepsilon \\
-\varepsilon & -2 \varepsilon \gamma_{0}
\end{array}\right]\left[\begin{array}{c}
v_{0} \\
(S v)_{0}
\end{array}\right] \\
& +\left[\begin{array}{c}
v_{N} \\
(S v)_{N}
\end{array}\right]^{T}\left[\begin{array}{cc}
-a+2 \tau_{N} & \varepsilon \\
\varepsilon & -2 \varepsilon \gamma_{N}
\end{array}\right]\left[\begin{array}{c}
v_{N} \\
(S v)_{N}
\end{array}\right],
\end{aligned}
$$

where $\tilde{R}=R-\gamma_{0} E_{0}+\gamma_{N} E_{N}$. To make (6) stable we need $\tilde{R} \geq 0$ and also that the two quadratic forms in (11) are negative semi-definite. This will be fulfilled if

$$
\tau_{0} \leq-a / 2-\varepsilon /\left(4 \Delta x_{1}\right), \quad \tau_{N} \leq a / 2-\varepsilon /\left(4 \Delta x_{N}\right) .
$$

These stability requirements are derived in Appendix A.

\section{Numerical experiments}

The partial differential equation (PDE) is well-posed and the numerical scheme is stable. The next question is: How accurate will the results be when doing simulations? We begin be looking and the hyperbolic and parabolic problems separately. At steady-state the time derivative is zero, and we mimic this by numerically implementing the ODE's

$$
\begin{array}{rlrl}
u_{x} & =F_{1}(x), & u(0) & =g_{0} \\
-u_{x x} & =F_{2}(x), & & \\
& u(0)=g_{0}, & u(1)=g_{1}
\end{array}
$$

in the domain $0 \leq x \leq 1$. We consider the manufactured solution $u(x)=\sin (5 \pi x / 2)+$ $x^{2}+1$. Consequently $F_{1}=5 \pi \cos (5 \pi x / 2) / 2+2 x$ and $F_{2}=(5 \pi)^{2} \sin (5 \pi x / 2) / 4-2$. (The solution $u$ was chosen in order to not be $2 \pi$-periodic or having zero valued derivatives on the boundaries.)

A discrete version of (13) and (14) becomes

$$
\begin{aligned}
P^{-1} Q v & =F_{1}+\tau_{0} P^{-1} e_{0}\left(v_{0}-g_{0}\right) \\
-P^{-1} M v & =F_{2}+\tau_{0} P^{-1} e_{0}\left(v_{0}-g_{0}\right)+\tau_{N} P^{-1} e_{N}\left(v_{N}-g_{N}\right)
\end{aligned}
$$


where $M=-A+B S$. Equation (15) and (16) was implemented using the stability requirements on $\tau_{0}$ and $\tau_{N}$ in (12), and solved using five differently designed grids.

The grids are specified in terms of the primal and dual mesh. The primal mesh consists of the integer-index solution points and the dual mesh of the flux points, see Figure 1. For the primal mesh we will require the condition stated below

Assumption 3.1. Assume that all $\Delta x_{i}$ have the same probability distribution (this restriction excludes stretched meshes). Hence

$$
E\left(\Delta x_{i}\right)=1 / N, \quad E\left(\left(\Delta x_{i}\right)^{p}\right)=m_{p} / N^{p}
$$

where $N$ is the number of grid points and the constant $m_{p}$ is dependent on the probability distribution chosen. For a deterministic mesh such that $\Delta x_{i} \equiv 1 / N$ we have $m_{p} \equiv 1$.

For the primal grid the following notation will be used. If all $\Delta x_{i}=h=1 / N$ then the primal mesh is said to be 'equidistant' (or uniform). If the $\Delta x_{i}$ 's are randomly perturbed, the primal mesh is called 'random'. For the control volumes we have a corresponding notation. If the flux points $x_{i+1 / 2} \equiv\left(x_{i}+x_{i+1}\right) / 2$ the dual mesh will be called 'centered'. If $x_{i+1 / 2}$ is perturbed by a constant we call the dual mesh 'shifted' and if the flux points are perturbed randomly we call it 'random'.

The discretization errors are defined as $e_{i}=u\left(x_{i}\right)-v_{i}$ and the truncation errors as $T_{e}=P^{-1} Q u-F_{1}$ and $T_{e}=-P^{-1} M u-F_{2}$, respectively. The resulting convergence rates for $e$ and $T_{e}$ for the two cases are given in Table 1 and Table 2. In Figure 2 and Figure 3 the $L_{2}$-norms of $e$ are plotted. The slopes in the figures correspond to the rates listed in the tables.

\begin{tabular}{|c|c|c|c|c|c|}
\hline Primal mesh: & uniform & uniform & uniform & random & random \\
\hline Dual mesh: & centered & shifted & random & centered & random \\
\hline Rate of Conv. $L_{2}(e)$ & 2 & 1 & 0.5 & 1.5 & 0.5 \\
Rate of Conv. $L_{2}\left(T_{e}\right)$ & 1.5 & 0.5 & 0 & 1 & 0 \\
R.o.C. $L_{2}\left(T_{e}\right)$ (inner points) & 2 & 2 & 0 & 1 & 0 \\
\hline
\end{tabular}

Table 1: Hyperbolic case. Order of accuracy for $e=u-v$ when solving $u_{x}=F_{1}$.

\begin{tabular}{|c|c|c|c|c|c|}
\hline Primal mesh: & uniform & uniform & uniform & random & random \\
\hline Dual mesh: & centered & shifted & random & centered & random \\
\hline Rate of Conv. $L_{2}(e)$ & 2 & 2 & 1.5 & 2 & 1.5 \\
Rate of Conv. $L_{2}\left(T_{e}\right)$ & 0.5 & 0.5 & 0 & 0.5 & 0 \\
R.o.C. $L_{2}\left(T_{e}\right)$ (inner points) & 2 & 2 & 0 & 1 & 0 \\
\hline
\end{tabular}

Table 2: Elliptic case. Order of accuracy for $e=u-v$ when solving $-u_{x x}=F_{2}$.

One can conclude that a centering of the dual mesh, i.e. to have $x_{i+1 / 2} \equiv\left(x_{i}+x_{i+1}\right) / 2$, always gives the best rate of convergence. Another observation is that the order of accuracy of the truncation error does not seem to have a clear relation to that of the discretization error, see Table 1 and 2 . This was also the observation in [2], [14].

Remark: Solving with randomly distributed grid points naturally gives rise to random errors. Therefore we have for those meshes (i.e. 'equidistant, random', 'random, 
centered' and 'random, random') made 500 simulation runs. In the figures the mean of the $500 L_{2}$-norms of the discretization errors are plotted.

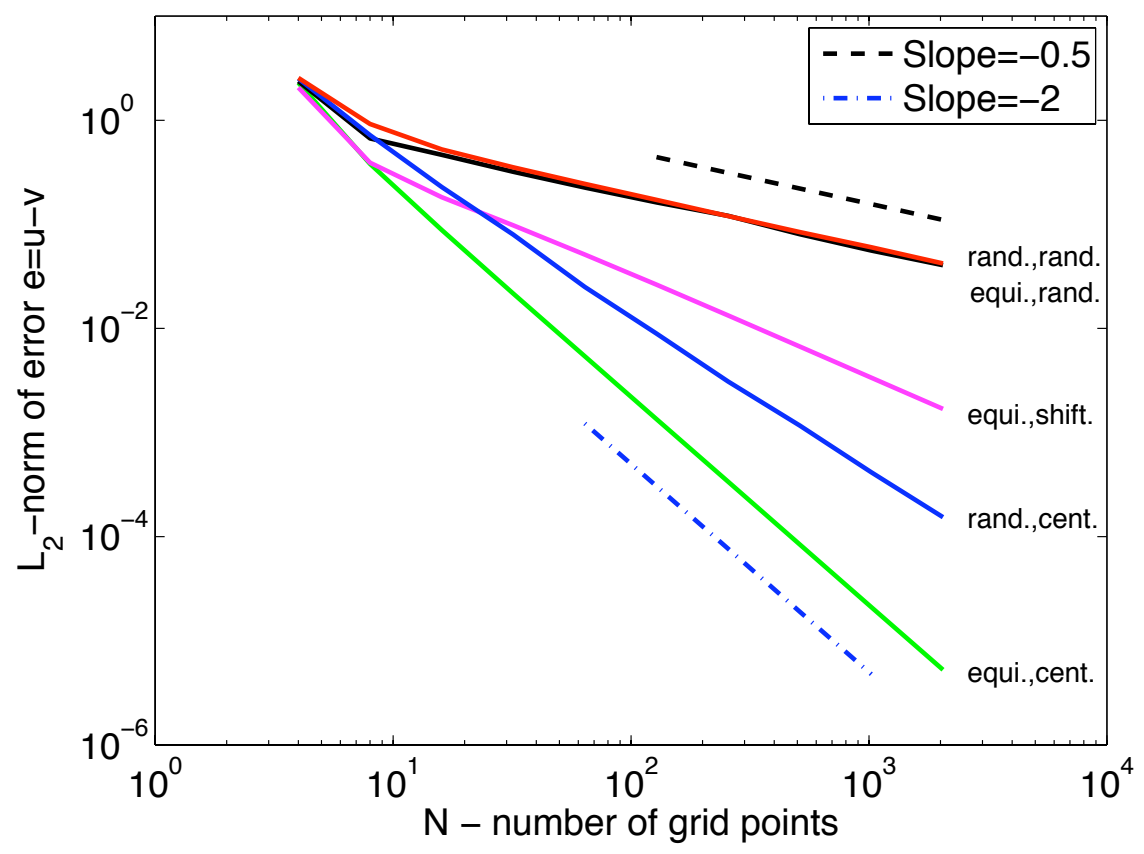

Figure 2: Hyperbolic case. Discretization error $e=u-v$ as a function of number of gridpoints $N$, when solving $u_{x}=F_{1}$. For the random grids a mean value after 500 test runs is shown.

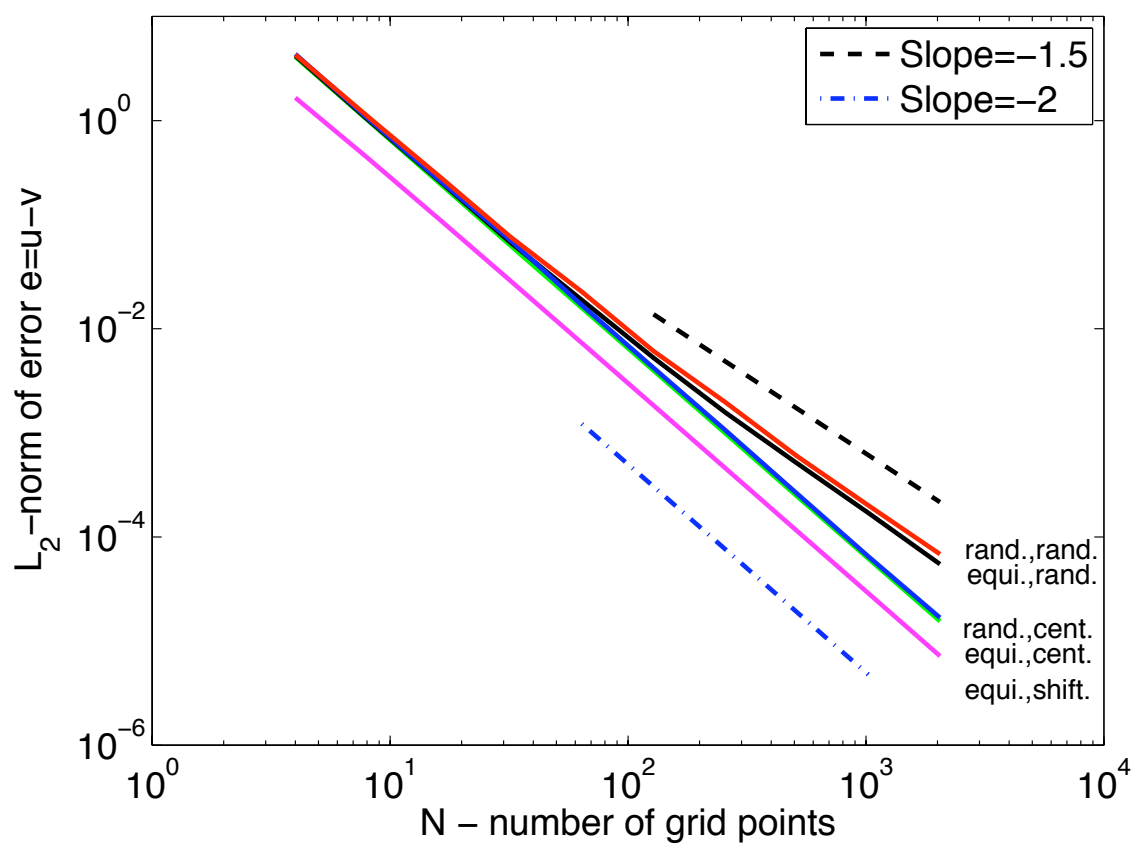

Figure 3: Parabolic case. Discretization error $e=u-v$ as a function of number of gridpoints $N$, when solving $-u_{x x}=F_{2}$. For the random grids a mean value after 500 test runs is shown. 


\section{Order of accuracy in the hyperbolic case}

To find out how the discretization errors depend on the truncation errors, we analyze the model problems further. The discrete formulation of the hyperbolic problem in (13) is given in (15) where $v_{i}$ is the numerical approximation of $u\left(x_{i}\right)$. Let $\tilde{Q}=Q-\tau_{0} E_{0}$ and rewrite (15) as

$$
v=\tilde{Q}^{-1}\left(P F-\tau_{0} e_{0} g\right) .
$$

The truncation error $T_{e}=P^{-1} \tilde{Q} u-F+\tau_{0} P^{-1} e_{0} g$ is obtained by inserting the exact solution $u$ into (15). That leads to

$$
u=\tilde{Q}^{-1}\left(P F-\tau_{0} e_{0} g+P T_{e}\right) .
$$

Combining equation (18) and (19), we obtain

$$
e=u-v=\tilde{Q}^{-1} P T_{e}
$$

$\tilde{Q}$ is non-singular if $\tau_{0} \neq 0$, and is inverted explicitly in Appendix B. The result is

$$
\tilde{Q}^{-1}=\frac{1}{\tau_{0}}\left[\begin{array}{rrrrrrr}
-1 & 1 & -1 & 1 & & 1 & -1 \\
-1 & 1 & -1 & 1 & & 1 & -1 \\
-1 & 1 & -1 & 1 & & 1 & -1 \\
-1 & 1 & -1 & 1 & & 1 & -1 \\
& & \vdots & & & & \vdots \\
-1 & 1 & -1 & 1 & & 1 & -1 \\
-1 & 1 & -1 & 1 & & 1 & -1
\end{array}\right]+2\left[\begin{array}{rrrrrrr}
0 & 0 & 0 & 0 & & 0 & 0 \\
0 & 1 & -1 & 1 & & 1 & -1 \\
0 & 1 & 0 & 0 & & 0 & 0 \\
0 & 1 & 0 & 1 & & 1 & -1 \\
& & \vdots & & & & \vdots \\
0 & 1 & 0 & 1 & & 1 & -1 \\
0 & 1 & 0 & 1 & \cdots & 1 & 0
\end{array}\right] .
$$

Note that $\tilde{Q}^{-1}$ has one constant part and one part that varies from row to row.

\subsection{Computation of the discretization error}

The point-wise discretization error $e_{i}=\left(\tilde{Q}^{-1} P T_{e}\right)_{i}$ can now be split into two parts as

$$
e_{i}=\frac{1}{\tau_{0}} e_{\tau}+2 \tilde{e}_{i}
$$

where $e_{\tau}=-\sum_{j=0}^{N}(-1)^{j}\left(P T_{e}\right)_{j}$ is constant in all grid points. The error part $\tilde{e}_{i}$ is more complex and varies with the index $i$. It is given in Appendix C.

To compute $e_{\tau}$ we need $P T_{e}$. First define $x_{i-1 / 2}=\left(x_{i-1}+x_{i}\right) / 2-\xi_{i}$, where $\left|\xi_{i}\right|<$ $\Delta x_{i} / 2$. (I.e. $\xi_{i}$ introduces an arbitrary perturbation around a centered dual mesh). The control volumes then become

$$
\Omega_{i}=\frac{\Delta x_{i+1}+\Delta x_{i}}{2}-\xi_{i+1}+\xi_{i}, \quad \Omega_{0}=\frac{\Delta x_{1}}{2}-\xi_{1}, \quad \Omega_{N}=\frac{\Delta x_{N}}{2}+\xi_{N} .
$$


We Taylor expand $P T_{e}=Q u-P F$ and denote $F^{i} \equiv F\left(x_{i}\right)$. Recalling $(7)$ and inserting $u_{x}=F$ and $u_{x x}=F_{x}$, we can write

$$
\begin{aligned}
& \left(P T_{e}\right)_{0}=\xi_{1} F^{0}+\frac{\Delta x_{1}^{2}}{4} F_{x}^{0}+\mathcal{O}\left(h^{3}\right) \\
& \left(P T_{e}\right)_{i}=\left(\xi_{i+1}-\xi_{i}\right) F^{i}+\left(\frac{\Delta x_{i+1}^{2}-\Delta x_{i}^{2}}{4}\right) F_{x}^{i}+\mathcal{O}\left(h^{3}\right) \\
& \left(P T_{e}\right)_{N}=-\xi_{N} F^{N}-\frac{\Delta x_{N}^{2}}{4} F_{x}^{N}+\mathcal{O}\left(h^{3}\right)
\end{aligned}
$$

which yields

$$
e_{\tau}=\underbrace{\sum_{i=1}^{N}(-1)^{i} \xi_{i}\left(F^{i-1}+F^{i}\right)}_{e_{\xi}}+\underbrace{\frac{1}{4} \sum_{i=1}^{N}(-1)^{i} \Delta x_{i}^{2}\left(F_{x}^{i-1}+F_{x}^{i}\right)}_{e_{\Delta x}}+\mathcal{O}\left(h^{2}\right) .
$$

Note that $e_{\xi}$ depends on the dual mesh and $e_{\Delta x}$ on the primal mesh.

Remark: Since the error can be divided into a dual mesh dependent part an a primal mesh dependent part, one can analyze the error contribution from the dual and primal mesh separately, rather than analyzing all combination of grids.

\subsection{Dual mesh dependent error}

First consider the dual mesh (computation of $e_{\xi}$ ). When the control volumes are centered then $\xi_{i}=0$ and therefore $e_{\xi}=0$. If the dual mesh is shifted $\left(\xi_{i}=C h\right)$ cancellation yields $e_{\xi}=C h\left(F^{N}-F^{0}\right)$ which means that an $\mathcal{O}(h)$ contribution is added to the discretization error.

The random case requires more explanation. The expected value of $e_{\xi}, E\left(e_{\xi}\right)$, will be equal to zero if $E\left(\xi_{i}\right)=0$. On the other hand, the "worst case scenario" when one considers $\xi_{i} \sim \mathcal{O}(h)$, leads to $e_{\xi}$ being $\mathcal{O}(1)$. The deviation from the mean value, $\sigma\left(e_{\xi}\right)$, will give the correct answer from a statistical point of view.

Proposition 4.1. Consider solving the problem (13) using (15) on a mesh where the control volumes are randomly distributed, so that $\xi_{i}=k r_{i} \Delta x_{i}$ and where Assumption 3.1 holds. Assume that the random variable $r_{i}$ is uniformly distributed in $[-1,1]$ and that $|k|<1 / 2$ is a constant. Then we have

$$
E\left(e_{\xi}\right)=0, \quad \sigma\left(e_{\xi}\right) \sim \mathcal{O}\left(h^{0.5}\right)
$$

where $E\left(e_{\xi}\right)$ is the expected value of $e_{\xi}$ and $\sigma\left(e_{\xi}\right)$ the standard deviation. Hence the contribution from the dual mesh to the discretization error is on average $e_{\xi} \sim \mathcal{O}\left(h^{0.5}\right)$.

Proof of proposition 4.1. The relation $\xi_{i}=k r_{i} \Delta x_{i}$ inserted into (25) gives

$$
e_{\xi}=k \sum_{i=1}^{N}(-1)^{i} r_{i} \Delta x_{i}\left(F^{i-1}+F^{i}\right)
$$


The mean of $e_{\xi}, E\left(e_{\xi}\right)$, is zero since $E\left(r_{i}\right)=0$. To compute the standard deviation $\sigma\left(e_{\xi}\right)$ we need

$$
e_{\xi}^{2}=k^{2}\left(\sum_{i=1}^{N} r_{i}^{2} \Delta x_{i}^{2}\left(F^{i-1}+F^{i}\right)^{2}+\sum_{i \neq j}(-1)^{i+j} r_{i} r_{j} \Delta x_{i} \Delta x_{j}\left(F^{i-1}+F^{i}\right)\left(F^{j-1}+F^{j}\right)\right)
$$

and

$$
E\left(r_{i}^{2}\right)=\int_{-\infty}^{\infty} p\left(r_{i}\right) r_{i}^{2} d r=\int_{-1}^{1} \frac{1}{2} r_{i}^{2} d r=\frac{1}{3}, \quad E\left(r_{i} r_{j}\right)=E\left(r_{i}\right) E\left(r_{j}\right)=0 .
$$

Using (28), (29) and Assumption 3.1 we obtain

$$
E\left(e_{\xi}^{2}\right)=\frac{k^{2}}{3} \frac{m_{2}}{N^{2}} \underbrace{\sum_{i=1}^{N}\left(F^{i-1}+F^{i}\right)^{2}}_{\varphi N}=\frac{k^{2} m_{2} \varphi}{3 N}
$$

where $\varphi$ is an $\mathcal{O}(1)$ constant depending on the function $F$. (For example if $F=c_{0}$ then $\varphi=4 c_{0}^{2}$ and if $F=c_{1} x$ then $\varphi=c_{1}^{2}\left(4 / 3-\mathcal{O}\left(h^{2}\right)\right)$.) The standard deviation $\sigma\left(e_{\xi}\right)$ is obtained by taking the square root of the variance $\mathrm{V}$,

$$
\begin{aligned}
& V\left(e_{\xi}\right)=E\left(e_{\xi}^{2}\right)-E\left(e_{\xi}\right)^{2}=\frac{k^{2} m_{2} \varphi}{3 N} \\
& \sigma\left(e_{\xi}\right)=\sqrt{V\left(e_{\xi}\right)}=k \sqrt{\frac{m_{2} \varphi}{3 N}} \sim \mathcal{O}\left(h^{0.5}\right) .
\end{aligned}
$$

Hence the error contribution $e_{\xi}$ is on average $\mathcal{O}\left(h^{0.5}\right)$, when using random dual mesh.

The error contributions introduced by the dual meshes are summarized in Table 3.

\subsection{Primal mesh dependent error}

Now consider the primal mesh (the component $e_{\Delta x}$ in (25)). The equidistant case, i.e. $\Delta x_{i}=h$, is straight-forward. Due to cancellation $e_{\Delta x}=h^{2}\left(F_{x}^{N}-F_{x}^{0}\right) / 4$, and we conclude that an equidistant primal mesh contributes with an $\mathcal{O}\left(h^{2}\right)$ error.

The random case requires statistical analysis. For the simplest case $F_{x}=c_{1}$ we get $e_{\Delta x}=c_{1}\left(-\Delta x_{1}^{2}+\Delta x_{2}^{2}-\ldots+\Delta x_{N}^{2}\right) / 2$. At best, all $\Delta x_{i}$ 's have the same size and $e_{\Delta x}=0$, and at worst they vary such that $e_{\Delta x} \sim \mathcal{O}(h)$. The statistical result (for a general $F$ ) is given in Proposition 4.2.

Proposition 4.2. Consider solving the problem (13) using (15) on a mesh with randomly distributed primal mesh, such that Assumption 3.1 holds. Then we have

$$
E\left(e_{\Delta x}\right) \sim \mathcal{O}\left(h^{2}\right), \quad \sigma\left(e_{\Delta x}\right) \sim \mathcal{O}\left(h^{1.5}\right)
$$

i.e. the primal mesh contribution to the discretization error is on average $e_{\Delta x} \sim \mathcal{O}\left(h^{1.5}\right)$. 
Proof of proposition 4.2. Consider $e_{\Delta x}$ in (25). Assumption 3.1 leads to

$$
E\left(\Delta x_{i}^{2}\right)=\frac{m_{2}}{N^{2}}, \quad E\left(\Delta x_{i}^{4}\right)=\frac{m_{4}}{N^{4}}
$$

which yields $E\left(e_{\Delta x}\right)=m_{2}\left(F_{x}^{N}-F_{x}^{0}\right) /\left(4 N^{2}\right)$ due to cancellation. In order to compute the standard deviation we need

$$
e_{\Delta x}^{2}=\frac{1}{16} \sum_{i=1}^{N} \Delta x_{i}^{4}\left(F_{x}^{i-1}+F_{x}^{i}\right)^{2}+\frac{2}{16} \underbrace{\sum_{i \neq j}(-1)^{i+j} \Delta x_{i}^{2} \Delta x_{j}^{2}\left(F_{x}^{i-1}+F_{x}^{i}\right)\left(F_{x}^{j-1}+F_{x}^{j}\right)}_{\zeta}
$$

where $\zeta$ consists of $N(N-2) / 4$ terms with positive sign, and $N^{2} / 4$ negative terms. We use the relations in (33) to obtain

$$
\begin{aligned}
E\left(e_{\Delta x}^{2}\right) & =\frac{1}{16} \frac{m_{4}}{N^{4}} \underbrace{\sum_{i=1}^{N}\left(F_{x}^{i-1}+F_{x}^{i}\right)^{2}}_{\varphi_{1} N}-\frac{2}{16} \frac{m_{2}^{2}}{N^{4}} \underbrace{\sum_{i \neq j}\left(F_{x}^{i-1}+F_{x}^{i}\right)\left(F_{x}^{j-1}+F_{x}^{j}\right)}_{\varphi_{2} N / 2} \\
& =\frac{m_{4} \varphi_{1}-m_{2}^{2} \varphi_{2}}{16 N^{3}} .
\end{aligned}
$$

The constants $m_{2}$ and $m_{4}$ depends on how $\Delta x_{i}$ is distributed. The constants $\varphi_{1}$ and $\varphi_{2}$ depends on $F_{x}$, and if $F_{x}=c_{1}$ then $\varphi_{1}=\varphi_{2}=4 c_{1}^{2}$. The constants $m_{2}, m_{4}, \varphi_{1}, \varphi_{2}$ will automatically be such that (35) is positive, since we are computing the mean value of a square. Hence we can put $K=\sqrt{m_{4} \varphi_{1}-m_{2}^{2} \varphi_{2}}$. Then

$$
\sigma\left(e_{\Delta x}\right)=\sqrt{E\left(e_{\Delta x}^{2}\right)-E\left(e_{\Delta x}\right)^{2}}=\frac{K}{4 \sqrt{N^{3}}}+\mathcal{O}\left(h^{2.5}\right) .
$$

Thus a random primal mesh gives an $\mathcal{O}\left(h^{1.5}\right)$ error contribution since $\sigma\left(e_{\Delta x}\right) \sim 1 / N^{1.5}$.

\subsection{Result of the analysis of the hyperbolic case}

In (22) we have split the discretization error $e$ into one constant part $e_{\tau}$ and one varying part $\tilde{e}$. We divide $\tilde{e}_{i}$ into a dual mesh dependent and a primal mesh dependent part just as we did with $e_{\tau}$

$$
\tilde{e}_{i}=\tilde{e}_{\xi}+\tilde{e}_{\Delta x}+\mathcal{O}\left(h^{2}\right)
$$

and perform similar analysis as in the previous sections. This is done in Appendix $\mathrm{C}$ and the result is listed in table 3 and 4 . From the tables it is clear that $e_{\tau}$ and $\tilde{e}_{i}$ have the same order of accuracy.

Remark: The contribution from $e_{\tau}$ to the discretization error $e$ is equal in every grid point. $\tilde{e}$ on the other hand varies from grid point to grid point. In particular, $\tilde{e}_{0}=0$. If $\tau_{0} \rightarrow \infty$ then $e_{0} \rightarrow 0$, i.e. with increased penalty the numerical solution $v_{0}$ tends to the exact solution $u(0)$. However, in the rest of the domain one can not (in the general case) say whether increased penalty leads to small errors. 


\begin{tabular}{|l|c|c|c|c|}
\hline \multicolumn{2}{|c|}{ Dual mesh } & \multicolumn{2}{c|}{ Error part } & Collective \\
\hline Notation & Form of $\xi_{i}$ & $e_{\xi}$ & $\tilde{e}_{\xi}$ & Order of acc. \\
\hline Centered & 0 & 0 & 0 & $\infty$ \\
Shifted & $C h$ & $C h\left(F^{N}-F^{0}\right)$ & $\frac{1-(-1)^{i}}{2} C h F^{N}$ & 1 \\
Random & $k \Delta x_{i} r_{i}$ & $\sigma\left(e_{\xi}\right) \sim k h^{0.5}$ & $\sigma\left(\tilde{e}_{\xi}\right) \sim k h^{0.5}$ & 0.5 \\
\hline
\end{tabular}

Table 3: Error contribution from the dual mesh when solving a hyperbolic problem.

\begin{tabular}{|l|c|c|c|c|}
\hline \multicolumn{2}{|c|}{ Primal mesh } & \multicolumn{2}{c|}{ Error part } & Collective \\
\hline Notation & Form of $\Delta x_{i}$ & $e_{\Delta x}$ & $\tilde{e}_{\Delta x}$ & Order of acc. \\
\hline Equidist. & $h$ & $h^{2}\left(F_{x}^{N}-F_{x}^{0}\right) / 4$ & $\frac{1-(-1)^{i}}{2} h^{2} F_{x}^{N} / 4$ & 2 \\
Random & $E\left(\Delta x_{i}\right)=h$ & $\sigma\left(e_{\Delta x}\right) \sim h^{1.5}$ & $\sigma\left(\tilde{e}_{\Delta x}\right) \sim h^{1.5}$ & 1.5 \\
\hline
\end{tabular}

Table 4: Error contribution from the primal mesh when solving a hyperbolic problem.

As we can see from table 5 the results from the analysis agree perfectly with the rates of convergence obtained experimentally. This is due to the fact that the inverse of the discrete operator, i.e. the direct link between the truncation error and the discretization error, can be computed exactly.

\begin{tabular}{|c|c|c|c|c|c|}
\hline Primal mesh: & uniform & uniform & uniform & random & random \\
\hline Dual mesh: & centered & shifted & random & centered & random \\
\hline $\begin{array}{c}\text { R.o.C. } L_{2}(e) \\
\text { Analysis }\end{array}$ & 2 & 1 & 0.5 & 1.5 & 0.5 \\
$\min (\infty, 2)$ & $\min (1,2)$ & $\min (0.5,2)$ & $\min (\infty, 1.5)$ & $\min (0.5,1.5)$ \\
\hline
\end{tabular}

Table 5: Hyperbolic case. Order of accuracy for $e=u-v$ when solving $u_{x}=F_{1}$.

Note that $\Delta x_{i} \sim 1 / N$ is assumed. For a linearly stretched mesh such that $\Delta x_{i}=k \Delta x_{i-1}$ this is not the case, since $\Delta x_{i}=k^{i-1}(k-1) /\left(k^{N}-1\right)$ is not proportional to $1 / N$.

\section{Order of accuracy in the elliptic case}

Next, we consider the case with second derivatives. Equation (14) in discretized form is given in (16). Let $\tilde{M}=M+\tau_{0} E_{0}+\tau_{N} E_{N}$ and rewrite (16) as

$$
v=-\tilde{M}^{-1}\left(P F-\tau_{0} e_{0} g_{0}-\tau_{N} e_{N} g_{N}\right) .
$$

Analogous to the hyperbolic case we get

$$
e=-\tilde{M}^{-1} P T_{e} .
$$


In order to express the discretization error $e$ in terms of the truncation error $T_{e}$ we need the inverse of $\tilde{M} . \tilde{M}$ is inverted exactly in Appendix D, and the result is

$$
\begin{aligned}
\left(\tilde{M}^{-1}\right)_{i 0}=\frac{1}{\tau_{0}} \sum_{k=i+1}^{N} \Delta x_{k}, \quad\left(\tilde{M}^{-1}\right)_{i N}=\frac{1}{\tau_{N}} \sum_{k=1}^{i} \Delta x_{k} \\
\text { For } i \geq j: \quad\left(\tilde{M}^{-1}\right)_{i j}=-\sum_{k=1}^{j} \Delta x_{k} \sum_{k=i+1}^{N} \Delta x_{k} \\
\text { For } i \leq j: \quad\left(\tilde{M}^{-1}\right)_{i j}=-\sum_{k=1}^{i} \Delta x_{k} \sum_{k=j+1}^{N} \Delta x_{k}
\end{aligned}
$$

The row index $i$ goes from 0 to $N$, and so does the column index $j$. On the diagonal (41) and (42) coincide.

\subsection{Computation of the discretization error}

We Taylor expand $P T_{e}=-M u-P F$ and insert $-u_{x x}=F,-u_{x x x}=F_{x}$. Denote $F^{i} \equiv F\left(x_{i}\right)$ and use $\Omega_{0}=\frac{\Delta x_{1}}{2}-\xi_{1}$ and $\Omega_{N}=\frac{\Delta x_{N}}{2}+\xi_{N}$ from (23). That leads to

$$
\begin{aligned}
\left(P T_{e}\right)_{0} & =\left(\xi_{1}-\frac{\Delta x_{1}}{2}\right) F^{0} \\
\left(P T_{e}\right)_{i} & =\left(\xi_{i+1}-\xi_{i}\right) F^{i}+\left(\frac{\Delta x_{i+1}^{2}-\Delta x_{i}^{2}}{6}\right) F_{x}^{i}+\mathcal{O}\left(h^{3}\right) \\
\left(P T_{e}\right)_{N} & =-\left(\xi_{N}+\frac{\Delta x_{N}}{2}\right) F^{N} .
\end{aligned}
$$

The error $e_{i}=-\left(\tilde{M}^{-1} P T_{e}\right)_{i}$ is computed and divided into one dual mesh dependent part and three primal mesh dependent parts.

$$
e_{i}=e_{\xi}+e_{\Delta_{x}}+\mathcal{O}\left(h^{2}\right)
$$

where

$$
\begin{aligned}
e_{\xi} & =\sum_{j=1}^{N} \xi_{j}\left(\left(\tilde{M}^{-1}\right)_{i j} F^{j}-\left(\tilde{M}^{-1}\right)_{i j-1} F^{j-1}\right) \\
e_{\Delta_{x}} & =\underbrace{\left(\tilde{M}^{-1}\right)_{i 0} \frac{\Delta x_{1}}{2} F^{0}}_{e_{i 0}}+\underbrace{\left(\tilde{M}^{-1}\right)_{i N} \frac{\Delta x_{N}}{2} F^{N}}_{e_{i N}}+\underbrace{\sum_{j=1}^{N-1}\left(\tilde{M}^{-1}\right)_{i j}\left(\frac{\Delta x_{j}^{2}-\Delta x_{j+1}^{2}}{6}\right) F_{x}^{j}}_{\tilde{e}_{\Delta x}}
\end{aligned}
$$

Here the error contribution $e_{\xi}$ is dependent on the dual grid, whereas $e_{i 0}, e_{i N}$ and $\tilde{e}_{\Delta x}$ depends on the primal grid.

Remark: Remember that we consider Dirichlet boundary conditions. For this particular boundary condition the stability requirement on the penalty parameters is that $\tau_{0} \leq-1 /\left(4 \Delta x_{1}\right)$ and $\tau_{N} \leq-1 /\left(4 \Delta x_{N}\right)$ should hold, see equation (12). This means that $\tau_{0, N}$ should be proportional to $N$. 


\subsection{Dual mesh dependent error}

The dual mesh dependent error contribution $e_{\xi}$ is given in (47). For a centered dual mesh the contribution is $e_{\xi}=0$ since $\xi_{j}=0$. If the dual mesh is shifted $\left(\xi_{i}=C h\right)$ we get $e_{\xi}=C h\left(\left(\tilde{M}^{-1}\right)_{i N} F^{N}-\left(\tilde{M}^{-1}\right)_{i 0} F^{0}\right)$ due to cancellation. We have $\left(\tilde{M}^{-1}\right)_{i 0}=$ $\left(\sum_{k=i+1}^{N} \Delta x_{k}\right) / \tau_{0} \sim \mathcal{O}(1) / \tau_{0}$ so consequently $\left(\tilde{M}^{-1}\right)_{i 0} \sim \mathcal{O}(h)$. The same is found for $\left(\tilde{M}^{-1}\right)_{i N}$ and hence $e_{\xi} \sim \mathcal{O}\left(h^{2}\right)$ in the shifted grid case.

We continue by looking at the case with random dual mesh.

Proposition 5.1. Consider solving the problem (14) using (16) with the dual mesh being random, i.e. $\xi_{i}=k r_{i} \Delta x_{i}$, where $r_{i} \in[-1,1]$ is uniformly distributed and the constant $|k|<1 / 2$. Assume that Assumption 3.1 holds. Then the error contribution from the dual mesh $e_{\xi}$ has the following properties

$$
E\left(e_{\xi}\right)=0, \quad \sigma\left(e_{\xi}\right) \sim \mathcal{O}\left(h^{1.5}\right)
$$

i.e. a random dual mesh error contributes statistically with an 1.5 order error.

Proof of proposition 5.1. From (46) we have

$$
e_{\xi}=\sum_{j=1}^{N} \xi_{j}\left(G_{i j}-G_{i j-1}\right)
$$

where we have defined $G_{i j}=\left(\tilde{M}^{-1}\right)_{i j} F^{j}$. The expected value of $e_{\xi}$ equals zero, i.e. $E\left(e_{\xi}\right)=0$, since $E\left(\xi_{j}\right)=0$ and $G_{i j}$ being independent of $\xi_{j}$. To find the statistically most probable error we compute the standard deviation. We need

$$
e_{\xi}^{2}=\sum_{j=1}^{N} \xi_{j}^{2}\left(G_{i j}-G_{i j-1}\right)^{2}+\sum_{j \neq n} \xi_{j} \xi_{n}\left(G_{i j}-G_{i j-1}\right)\left(G_{i n}-G_{i n-1}\right)
$$

Using the relations from (29) we get $E\left(\xi_{j}^{2}\right)=k^{2} E\left(r_{j}^{2}\right) E\left(\Delta x_{j}^{2}\right)=k^{2} m_{2} /\left(3 N^{2}\right) \sim \mathcal{O}\left(h^{2}\right)$ and $E\left(\xi_{j} \xi_{n}\right)=0$. From Appendix E we have $\left(G_{i j}-G_{i j-1}\right)^{2} \sim \mathcal{O}\left(h^{2}\right)$. We obtain

$$
\begin{aligned}
& E\left(e_{\xi}^{2}\right)=\mathcal{O}\left(h^{3}\right) \\
& \sigma\left(e_{\xi}\right)=\sqrt{E\left(e_{\xi}^{2}\right)-E\left(e_{\xi}\right)^{2}}=\mathcal{O}\left(h^{1.5}\right) .
\end{aligned}
$$

Hence the dual mesh error is on average proportional to $\mathcal{O}\left(h^{1.5}\right)$.

\subsection{Primal mesh dependent error}

The primal mesh dependent error was split into three parts as $e_{\Delta_{x}}=e_{i 0}+e_{i N}+\tilde{e}_{\Delta x}$. First consider the boundary errors $e_{i 0}$ and $e_{i N}$ from (48). Recalling that $\tau_{0} \sim 1 / \Delta x_{1}$, we get

$$
e_{i 0}=\frac{1}{\tau_{0}}\left(\sum_{k=i+1}^{N} \Delta x_{k}\right) \frac{\Delta x_{1}}{2} F^{0} \sim \Delta x_{1}^{2}
$$


and in the same way $e_{i N} \sim \Delta x_{N}^{2}$.

We now take a look at $\tilde{e}_{\Delta x}$. If the mesh is equidistant $\left(\Delta x_{i}=h\right)$, it is easily seen that $\tilde{e}_{\Delta x}=0$. For a general primal grid we can write

$$
\tilde{e}_{\Delta x}=\frac{\Delta x_{1}^{2}}{6} G_{i 1}^{\prime}-\frac{\Delta x_{N}^{2}}{6} G_{i N-1}^{\prime}+\underbrace{\sum_{j=2}^{N-1} \frac{\Delta x_{j}^{2}}{6}\left(G_{i j}^{\prime}-G_{i j-1}^{\prime}\right)}_{\zeta}
$$

where $G_{i j}^{\prime}$ is defined as $G_{i j}^{\prime}=\left(\tilde{M}^{-1}\right)_{i j} F_{x}^{j}$. From Appendix E we have $\left(G_{i j}^{\prime}-G_{i j-1}^{\prime}\right) \sim$ $\mathcal{O}(h)$, and hence $\zeta \sim \mathcal{O}\left(h^{2}\right)$. Furthermore $G_{i 1}^{\prime}$ and $G_{i N-1}^{\prime}$ are proportional to $\mathcal{O}(h)$. Thus the error contribution from $\tilde{e}_{\Delta x}$ will never be worse than $\mathcal{O}\left(h^{2}\right)$, and we conclude that the same holds for $e_{\Delta_{x}}$.

\subsection{Result of the analysis for the elliptic case}

The error contribution from the primal mesh was at worst $\mathcal{O}\left(h^{2}\right)$ which is the best possible result.

The main part of the error comes from the positioning of the flux points. Except if the control volumes are centered, since then there is no additional error introduced by the dual mesh. The dual mesh dependent errors are summarized in Table 6.

\begin{tabular}{|l|c|c|c|}
\hline \multicolumn{2}{|c|}{ Dual mesh } & Error part & Order \\
\hline Notation & Form of $\xi_{i}$ & $e_{\xi}$ & of accuracy \\
\hline Centered & 0 & 0 & $\infty$ \\
Shifted & $C h$ & $\sim C h^{2}$ & 2 \\
Random & $k \Delta x_{i} r_{i}$ & $\sigma\left(e_{\xi}\right) \sim k h^{1.5}$ & 1.5 \\
\hline
\end{tabular}

Table 6: Error contribution from the dual mesh when solving a hyperbolic problem.

In table 7 the numerical results shown earlier are listed together with the result from the analysis. Apparently they are in full agreement.

\begin{tabular}{|c|c|c|c|c|c|}
\hline Primal mesh: & uniform & uniform & uniform & random & random \\
\hline Dual mesh: & centered & shifted & random & centered & random \\
\hline R.o.C. & 2 & 2 & 1.5 & 2 & 1.5 \\
Analysis & $\min (\infty, 2)$ & $\min (2,2)$ & $\min (1.5,2)$ & $\min (\infty, 2)$ & $\min (1.5,2)$ \\
\hline
\end{tabular}

Table 7: Elliptic case. Order of accuracy for $e=u-v$ when solving $-u_{x x}=F_{2}$.

\section{Advection-diffusion equation}

Having analyzed the hyperbolic and elliptic parts separately, it is interesting to see how they combine. One common application for FVM computations is to solve the Navier-Stokes equations where both the hyperbolic and elliptic operators are involved. 
The corresponding one-dimensional model problem in the steady case is written

$$
a u_{x}=\varepsilon u_{x x}+F(x), \quad u(0)=g_{0}, \quad u(1)=g_{1},
$$

which we can solve by implementing the discrete formulation

$$
a P^{-1} Q v=\varepsilon P^{-1} M v+F+\tau_{0} P^{-1} e_{0}\left(v_{0}-g_{0}\right)+\tau_{N} P^{-1} e_{N}\left(v_{N}-g_{N}\right) .
$$

The truncation error $T_{e}$ is obtained by putting the exact solution $u$ into the numerical scheme. We write $P T_{e}=a Q u-\varepsilon M u-P F$ which using Taylor expansion becomes

$$
\begin{aligned}
\left(P T_{e}\right)_{0} & =\xi_{1} F^{0}+\varepsilon \frac{\Delta x_{1}}{2} u_{x x}^{0}+\mathcal{O}\left(h^{2}\right) \\
\left(P T_{e}\right)_{i} & =\left(\xi_{i+1}-\xi_{i}\right) F_{i}+\left(\Delta x_{i+1}^{2}-\Delta x_{i}^{2}\right)\left(\frac{a}{4} u_{x x}^{i}-\frac{\varepsilon}{6} u_{x x x}^{i}\right)+\mathcal{O}\left(h^{3}\right) \\
\left(P T_{e}\right)_{N} & =-\xi_{N} F_{N}+\varepsilon \frac{\Delta x_{N}}{2} u_{x x}^{N}+\mathcal{O}\left(h^{2}\right)
\end{aligned}
$$

Hence $\left(T_{e}\right)_{0}$ and $\left(T_{e}\right)_{N}$ are always are of order $\mathcal{O}(1)$, whereas the inner points $\left(T_{e}\right)_{i}$ have either order $\mathcal{O}(1), \mathcal{O}(h)$ or even $\mathcal{O}\left(h^{2}\right)$, depending on the function $F$ and the mesh.

To be more precise, for a 'uniform, centered' and a 'uniform, shifted' mesh we have an inner order of $\mathcal{O}\left(h^{2}\right)$, and for a 'uniform, random' mesh the order is $\mathcal{O}(F)+\mathcal{O}\left(h^{2}\right)$. If the mesh is 'random, centered' the order will be $\mathcal{O}(h)$ and if it is 'random, random' the order will be $\mathcal{O}(F)+\mathcal{O}(h)$. We call the order of the truncation error $p$ in the interior and $q$ at the boundaries, and get

$$
L_{2}\left(T_{e}\right) \sim \sqrt{h\left(h^{q}\right)^{2}+\sum_{i=1}^{N-1} h\left(h^{p}\right)^{2}} \sim \mathcal{O}\left(h^{\min (p, q+0.5)}\right) .
$$

So for a general $F$ we will have

\begin{tabular}{|c|c|c|c|c|c|c|c|}
\hline Primal mesh: & uniform & uniform & \multicolumn{2}{|c|}{ uniform } & random & \multicolumn{2}{c|}{ random } \\
\hline Dual mesh: & centered & shifted & \multicolumn{2}{|c|}{ random } & centered & \multicolumn{2}{c|}{ random } \\
\hline Forcing func. $F:$ & any & any & $F \neq 0$ & $F=0$ & any & $F \neq 0$ & $F=0$ \\
\hline $\mathrm{p}$ & 2 & 2 & 0 & 2 & 1 & 0 & 1 \\
$\mathrm{q}+0.5$ & 0.5 & 0.5 & 0.5 & 0.5 & 0.5 & 0.5 & 0.5 \\
R.o.C. $L_{2}\left(T_{e}\right)$ & 0.5 & 0.5 & 0 & 0.5 & 0.5 & 0 & 0.5 \\
\hline
\end{tabular}

Table 8: Advection-Diffusion. Order of accuracy for $e=u-v$ when solving $a u_{x}=\varepsilon u_{x x}+F$.

We conclude that for the two meshes with random dual mesh, the order of the truncation error will improve if the source function $F$ is zero. We examine this prediction by looking at the equation

$$
a u_{x}=\varepsilon u_{x x}, \quad u(0)=1, \quad u(1)=0
$$

which has the exact solution

$$
u(x)=\left(1-e^{a(x-1) / \varepsilon}\right) /\left(1-e^{-a / \varepsilon}\right) .
$$


Implementing and running numerical experiments using the five grids results in

\begin{tabular}{|c|c|c|c|c|c|}
\hline Primal mesh: & uniform & uniform & uniform & random & random \\
\hline Dual mesh: & centered & shifted & random & centered & random \\
\hline Rate of Conv. $L_{2}(e)$ & 2 & 2 & 2 & 2 & 2 \\
Rate of Conv. $L_{2}\left(T_{e}\right)$ & 0.5 & 0.5 & 0.5 & 0.5 & 0.5 \\
R.o.C. $L_{2}\left(T_{e}\right)$ (inner points) & 2 & 2 & 2 & 1 & 1 \\
\hline
\end{tabular}

Table 9: Advection-Diffusion. Order of accuracy for $e=u-v$ when solving $a u_{x}=\varepsilon u_{x x}$.

Note that all five grids gives a second order error. Having the forcing term equal to zero is of course a special case, but nevertheless an often used and important one. Keeping the forcing term non-zero gives exactly the same result as the elliptic case, see Table 2.

\begin{tabular}{|c|c|c|c|c|c|}
\hline Primal mesh: & uniform & uniform & uniform & random & random \\
\hline Dual mesh: & centered & shifted & random & centered & random \\
\hline Rate of Conv. $L_{2}(e)$ & 2 & 2 & 1.5 & 2 & 1.5 \\
Rate of Conv. $L_{2}\left(T_{e}\right)$ & 0.5 & 0.5 & 0 & 0.5 & 0 \\
R.o.C. $L_{2}\left(T_{e}\right)$ (inner points) & 2 & 2 & 0 & 1 & 0 \\
\hline
\end{tabular}

Table 10: Advection-Diffusion. Order of accuracy for $e=u-v$ when solving $a u_{x}=\varepsilon u_{x x}+F$.

Remark: We see that for the grids with random dual mesh the rate of convergence is improved by 0.5 if having the forcing function $F=0$. This can be understood by looking at the truncation error in (58), where $F_{i}$ is multiplied by $\xi_{i+1}-\xi_{i}$, i.e. the dual mesh perturbation.

\section{Summary}

The discrete versions of a hyperbolic and a elliptic differential equation with Dirichlet boundary conditions have been analyzed, and the numerical simulations agree perfectly with the analysis.

The corner stone in this analysis is that the discrete versions of the differential operators have been proven possible to invert. The fact that the errors introduced by random meshes are treated with probability theory is also of importance.

The grid design is clearly very important for the rate of convergence, especially for the hyperbolic case (the rate of convergence is 2 for a uniform mesh and 0.5 for a random mesh). For the elliptic problem the convergence rates found were 2 for a uniform mesh and at worst 1.5 for random meshes.

The most significant feature of a "good" grid is that the control volumes are centered, i.e. that the flux points are positioned right between the solution points. Note that this centering is very easy to create in one dimension, but it is not clear how to achieve the same thing in multiple dimensions.

We also solved the advection-diffusion problem with and without a forcing term. When the forcing term was included the results were exactly the same as for the elliptic 
case. Without any forcing term the solution was second order accurate for all grids. If this is applicable in multi dimensions this can be of great interest when solving for example the Navier-Stokes equations.

\section{A Penalty parameters for stability}

To guarantee that the numerical scheme in (6) is stable we have to make sure that $\tilde{R} \geq 0$ and that the two quadratic forms in (11) are negative semi-definite. The matrix $\tilde{R}=R-\gamma_{0} E_{0}+\gamma_{N} E_{N}$, where $R=S^{-T} A S^{-1}$, has the following structure

$$
\tilde{R}=\left[\begin{array}{ccccccc}
\Delta x_{1}-\gamma_{0} & 0 & & & & & \\
0 & \frac{1}{\Delta x_{2}} & \frac{-1}{\Delta x_{2}} & & & \\
0 & \frac{-1}{\Delta x_{2}} & \frac{1}{\Delta x_{2}}+\frac{1}{\Delta x_{3}} & \frac{-1}{\Delta x_{3}} & & & \\
& & \ddots & \ddots & \ddots & & \\
& & & \frac{-1}{\Delta x_{N-2}} & \frac{1}{\Delta x_{N-2}}+\frac{1}{\Delta x_{N-1}} & \frac{-1}{\Delta x_{N-1}} & 0 \\
& & & & \frac{1}{\Delta x_{N-1}} & \frac{1}{\Delta x_{N-1}} & 0 \\
& & & & & 0 & \Delta x_{N}-\gamma_{N}
\end{array}\right] .
$$

If a matrix is symmetric, diagonally dominant and real, with non-negative diagonal entries, then the matrix is positive definite. For $\tilde{R}$ this will be fulfilled if $\tilde{R}_{i i} \geq \sum_{j \neq i}\left|\tilde{R}_{i j}\right|$ for every row $i$, which means that if (63) holds, then $\tilde{R} \geq 0$.

$$
\Delta x_{1}-\gamma_{0} \geq 0 \quad \Delta x_{N}-\gamma_{N} \geq 0
$$

Further, the two quadratic forms in equation (11) are negative semi-definite if

$$
\begin{array}{rr}
a+2 \tau_{0} \leq 0 & -a+2 \tau_{N} \leq 0 \\
-2 \varepsilon \gamma_{0} \leq 0 & -2 \varepsilon \gamma_{N} \leq 0 \\
\varepsilon^{2} \leq\left(a+2 \tau_{0}\right)\left(-2 \varepsilon \gamma_{0}\right) & \varepsilon^{2} \leq\left(-a+2 \tau_{N}\right)\left(-2 \varepsilon \gamma_{N}\right)
\end{array}
$$

When implementing we have to choose the penalty parameters $\tau_{0}, \tau_{N}$ according to equation (64). We can treat both boundaries simultaneously with the following equations:

$$
\begin{array}{r}
-c a+2 \tau \leq 0 \\
-2 \varepsilon \gamma \leq 0 \\
\varepsilon^{2} \leq(-c a+2 \tau)(-2 \varepsilon \gamma)
\end{array}
$$

where $c=-1$ at the left boundary and $c=1$ at the right boundary. We identify two special cases. First, $\varepsilon=0$, the hyperbolic case: (65), (66) and (67) reduces to

$$
-c a+2 \tau \leq 0
$$

In the continuous counterpart of this case we have to bound the solution at the boundary where $c a \leq 0$. Therefore, if $c a>0$ we put $\tau=0$. If $c a<0$ we instead put $\tau \leq c a / 2$. Both scenarios are covered by

$$
\tau=k \frac{c a-|a|}{4}, \quad k \geq 1 .
$$


Secondly, $\varepsilon \neq 0$, the parabolic case: If we rewrite expression (67) using (66) we will get

$$
-c a+2 \tau \leq \frac{\varepsilon}{-2 \gamma} \leq 0
$$

hence expression (65) is unnecessary. Inserting $\gamma \leq \Delta x$ into equation (70) yields

$$
\tau \leq-\frac{\varepsilon}{4 \Delta x}+\frac{c a}{2}
$$

If $c a>0$ the penalty parameter $\tau$ may equal zero (even thought two boundary conditions are requiered). This can be solved by putting $\tau \leq-\varepsilon /(4 \Delta x)-|c a| / 2$.

Equation (69) in the hyperbolic case, or equation (71) in the parabolic case, gives the stability conditions on (6).

\section{B Derivation of $\tilde{Q}^{-1}$}

We have the difference operator $Q$ including the penalty parameter $\tau_{0}$ as

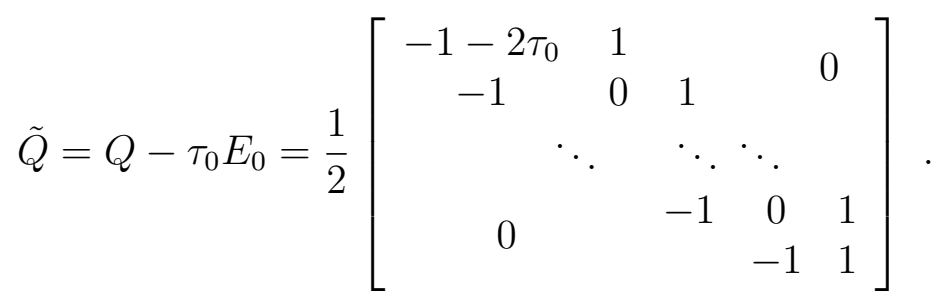

The inverse $\tilde{Q}^{-1}$ is derived using Gaussian elimination. Start by multiplying by 2 .

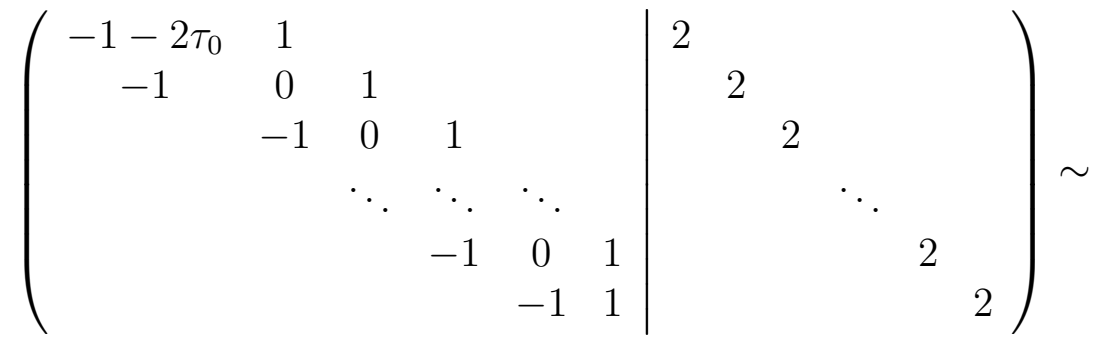

Subtract rows, starting at last row. Assume $N$ even, where $N+1$ is the number of rows.

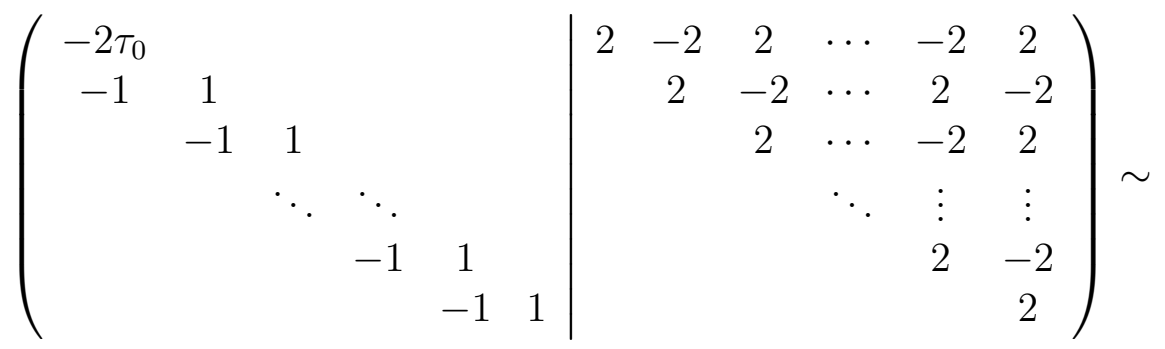

Define the help parameter $a=1 / \tau_{0}$ and write

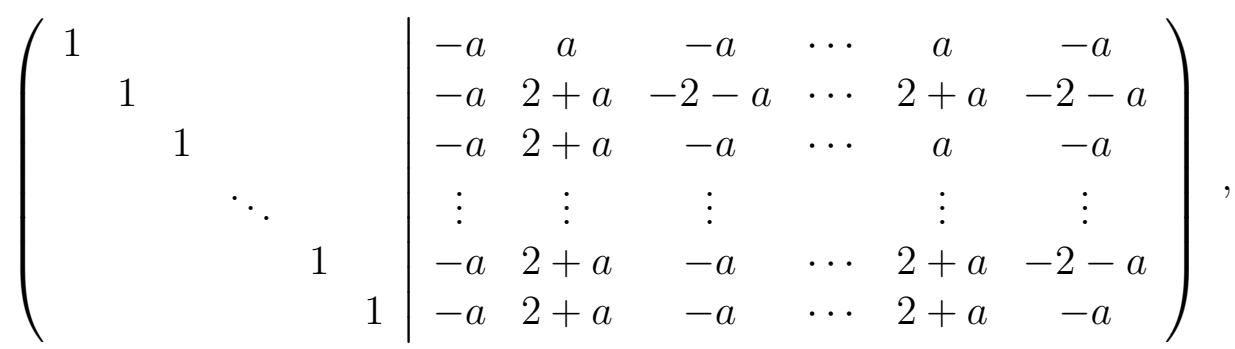


which, if going back to $\tau_{0}$, means that

$$
\tilde{Q}^{-1}=\frac{1}{\tau_{0}}\left[\begin{array}{cccccc}
-1 & 1 & -1 & & 1 & -1 \\
-1 & 1 & -1 & \cdots & 1 & -1 \\
-1 & 1 & -1 & & 1 & -1 \\
& \vdots & & & & \vdots \\
-1 & 1 & -1 & & 1 & -1 \\
-1 & 1 & -1 & & 1 & -1
\end{array}\right]+2\left[\begin{array}{cccccc}
0 & 0 & 0 & & 0 & 0 \\
0 & 1 & -1 & \cdots & 1 & -1 \\
0 & 1 & 0 & & 0 & 0 \\
& \vdots & & & & \vdots \\
0 & 1 & 0 & & 1 & -1 \\
0 & 1 & 0 & \cdots & 1 & 0
\end{array}\right] \text {. }
$$

Again, expression (76) assumes that $N$ is even, where $\tilde{Q}^{-1}$ is $(N+1) \times(N+1)$.

\section{Derivation of the hyperbolic error $\tilde{e}$}

In (22) the hyperbolic error is divided into one constant part $e_{\tau}$ and one part $\tilde{e}$ that varies over the grid points $i$. The varying error $\tilde{e}_{i}$ can be written

$$
\begin{array}{rlrl}
\tilde{e}_{i}^{\text {even }} & =\sum_{j=0}^{i} \frac{1-(-1)^{j}}{2}\left(P T_{e}\right)_{j}=\sum_{k=1}^{i / 2}\left(P T_{e}\right)_{2 k-1} & & \text { for even } i \\
\tilde{e}_{i}^{\text {odd }} & =\sum_{k=1}^{(i+1) / 2}\left(P T_{e}\right)_{2 k-1}-\sum_{j=i+1}^{N}(-1)^{j}\left(P T_{e}\right)_{j} & \text { for odd } i
\end{array}
$$

Just as $e_{\tau}$ the error $\tilde{e}$ can be divided into one dual mesh dependent and one primal mesh dependent part. Recalling (24) yields

$$
\tilde{e}_{i}^{\text {even }}=\underbrace{\sum_{k=1}^{i / 2}\left(\xi_{2 k}-\xi_{2 k-1}\right) F^{2 k-1}}_{\tilde{e}_{\xi}^{\text {even }}}+\underbrace{\sum_{k=1}^{i / 2}\left(\frac{\Delta x_{2 k}^{2}-\Delta x_{2 k-1}^{2}}{4}\right) F_{x}^{2 k-1}}_{\tilde{e}_{\Delta x}^{\text {even }}}+\mathcal{O}\left(h^{2}\right)
$$

and

$$
\begin{aligned}
\tilde{e}_{i}^{\text {odd }} & =\underbrace{\sum_{k=1}^{(i+1) / 2}\left(\xi_{2 k}-\xi_{2 k-1}\right) F^{2 k-1}-\sum_{j=i+1}^{N}(-1)^{j}\left(\xi_{j+1}-\xi_{j}\right) F^{j}}_{\tilde{e}_{\xi}^{\text {odd }}} \\
& +\underbrace{\sum_{k=1}^{(i+1) / 2}\left(\frac{\Delta x_{2 k}^{2}-\Delta x_{2 k-1}^{2}}{4}\right) F_{x}^{2 k-1}-\sum_{j=i+1}^{N}(-1)^{j}\left(\frac{\Delta x_{j+1}^{2}-\Delta x_{j}^{2}}{4}\right) F_{x}^{j}}_{\tilde{e}_{\Delta x}^{\text {odd }}}+\mathcal{O}\left(h^{2}\right) .
\end{aligned}
$$

First consider the dual mesh (computation of $\tilde{e}_{\xi}$ ). For $\xi_{i}=0$ we have $\tilde{e}_{\xi}^{\text {even }}=\tilde{e}_{\xi}^{\text {odd }}=0$. For $\xi_{i}=C h$ cancellation yields that $\tilde{e}_{\xi}^{\text {even }}=0$ and $\tilde{e}_{\xi}^{\text {odd }}=C h F^{N}$. Hence an $\mathcal{O}(h)$ error is added to the discretization error in every odd grid point $i$, in the shifted grid case. 
For the random dual mesh we assume the control volumes to be distributed such that $\xi_{i}=k r_{i} \Delta x_{i}$, where $r_{i} \in(-1,1)$ and that $|k|<1 / 2$. The relation in (79) is rewritten as

$$
\tilde{e}_{\xi}^{\text {even }}=k \sum_{j=1}^{i}(-1)^{j} r_{j} \Delta x_{j} F^{\varphi(j)}
$$

where $\varphi(j)=j-\left(1+(-1)^{j}\right) / 2$. The expected value $E\left(\tilde{e}_{\xi}^{e v e n}\right)=0$ since $E\left(r_{j}\right)=0$. We compute the deviation from the mean value, $\sigma\left(\tilde{e}_{\xi}^{\text {even }}\right)$ by

$$
\left(\tilde{e}_{\xi}^{\text {even }}\right)^{2}=k^{2}\left(\sum_{j=1}^{i} r_{j}^{2} \Delta x_{j}^{2}\left(F^{\varphi(j)}\right)^{2}+\sum_{n \neq j}(-1)^{n+j} r_{n} r_{j} \Delta x_{n} \Delta x_{j}\left(F^{\varphi(n)}\right)\left(F^{\varphi(j)}\right)\right) .
$$

From (29) we have $E\left(r_{j}^{2}\right)=1 / 3$ and $E\left(r_{n} r_{j}\right)=0$. Using Assumption 3.1 this yields

$$
E\left(\left(\tilde{e}_{\xi}^{\text {even }}\right)^{2}\right)=\frac{k^{2}}{3} \sum_{j=1}^{i} E\left(\Delta x_{j}^{2}\right)\left(F^{\varphi(j)}\right)^{2}=\frac{k^{2} m_{2} i \varphi}{3 N^{2}} \sim k^{2} \mathcal{O}(h)
$$

where $\varphi$ is a constant depending on $F$. The standard deviation $\sigma\left(\tilde{e}_{\xi}^{\text {even }}\right)$ is obtained by taking the square root of the variance. Hence we have

$$
E\left(\tilde{e}_{\xi}^{\text {even }}\right)=0 \quad \sigma\left(\tilde{e}_{\xi}^{\text {even }}\right) \sim k \mathcal{O}\left(h^{0.5}\right) .
$$

Combining this derivation with the methods in Proposition 4.1 will give us that the same result holds for $\tilde{e}_{\xi}^{o d d}$.

Next consider the primal mesh (computation of $\tilde{e}_{\Delta x}$ ). For $\Delta x_{i}=h$ we will have $\tilde{e}_{\Delta x}^{\text {even }}=0$ and $\tilde{e}_{\Delta x}^{o d d}=h^{2} F_{x}^{N} / 4$ due to cancellation, and we conclude that an equidistant primal mesh contributes with an $\mathcal{O}\left(h^{2}\right)$ error in every odd point $i$. For the random case we consider $\tilde{e}_{\Delta x}^{\text {even }}$ in (79) and $\tilde{e}_{\Delta x}^{\text {odd }}$ in (80). Using Assumption 3.1 we obtain $E\left(\tilde{e}_{\Delta x}^{e v e n}\right)=0$ whereas $E\left(\tilde{e}_{\Delta x}^{o d d}\right)=F_{x}^{N} m_{2} /\left(4 N^{2}\right)$. In order to compute the standard deviations we take the squares of $\tilde{e}_{\Delta x}^{e v e n}$ and get

$$
\left(\tilde{e}_{\Delta x}^{\text {even }}\right)^{2}=\frac{1}{16} \sum_{j=1}^{i} \Delta x_{j}^{4}\left(F_{x}^{\varphi(j)}\right)^{2}+\frac{2}{16} \underbrace{\sum_{n \neq j}(-1)^{n+j} \Delta x_{n}^{2} \Delta x_{j}^{2}\left(F_{x}^{\varphi(n)}\right)\left(F_{x}^{\varphi(j)}\right)}_{\zeta}
$$

where $\varphi(j)=j-\left(1+(-1)^{j}\right) / 2$. Here $\zeta$ consists of $i(i-2) / 4$ terms with positive sign and $i^{2} / 4$ negative terms. Using (33) we achieve

$$
E\left(\left(\tilde{e}_{\Delta x}^{\text {even }}\right)^{2}\right)=\frac{1}{16} \frac{m_{4}}{N^{4}} \sum_{j=1}^{i}\left(F_{x}^{\varphi(j)}\right)^{2}-\frac{2}{16} \frac{m_{2}^{2}}{N^{4}} \sum_{n \neq j}(-1)^{n+j}\left(F_{x}^{\varphi(n)}\right)\left(F_{x}^{\varphi(j)}\right)
$$

which we recognize from Proposition 4.2 to be proportinal to $i / N^{4} \sim \mathcal{O}\left(h^{3}\right)$. Using the same methods we will obtain $E\left(\left(\tilde{e}_{\Delta x}^{\text {odd }}\right)^{2}\right) \sim \mathcal{O}\left(h^{3}\right)$. Then

$$
\begin{aligned}
& \sigma\left(\tilde{e}_{\Delta x}^{\text {even }}\right)=\sqrt{\mathcal{O}\left(h^{3}\right)}=\mathcal{O}\left(h^{1.5}\right) \\
& \sigma\left(\tilde{e}_{\Delta x}^{\text {odd }}\right)=\sqrt{\mathcal{O}\left(h^{3}\right)-\mathcal{O}\left(h^{4}\right)}=\mathcal{O}\left(h^{1.5}\right) .
\end{aligned}
$$

Thus it holds that

$$
E\left(\tilde{e}_{\Delta x}\right) \sim \mathcal{O}\left(h^{2}\right) \quad \sigma\left(\tilde{e}_{\Delta x}\right) \sim \mathcal{O}\left(h^{1.5}\right)
$$

i.e. the primal mesh contribution to the discretization error is on average $\tilde{e}_{\Delta x} \sim \mathcal{O}\left(h^{1.5}\right)$. 


\section{Derivation of $\tilde{M}^{-1}$}

Define $c_{i}=1 / \Delta x_{i}$. Then we can write $\tilde{M}$ as

$$
\tilde{M}=\left[\begin{array}{ccccc}
\tau_{0} & & & & \\
c_{1} & -c_{1}-c_{2} & c_{2} & & \\
& \ddots & \ddots & \ddots & \\
& & c_{N-1} & -c_{N-1}-c_{N} & c_{N} \\
& & & & \tau_{N}
\end{array}\right]
$$

First, consider only the inner 1 to $N-1$ rows (ignore row 0 and $N$ for a while)

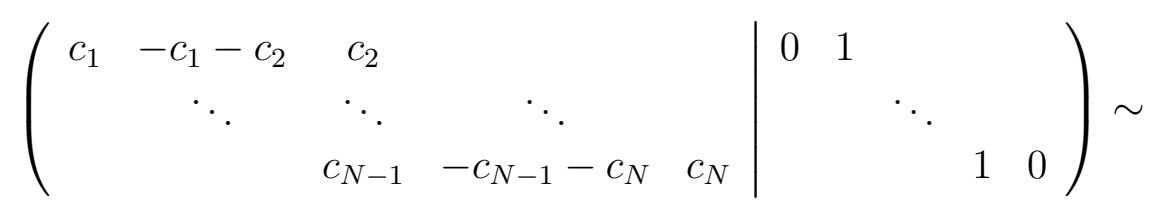

and start Gaussian elimination.

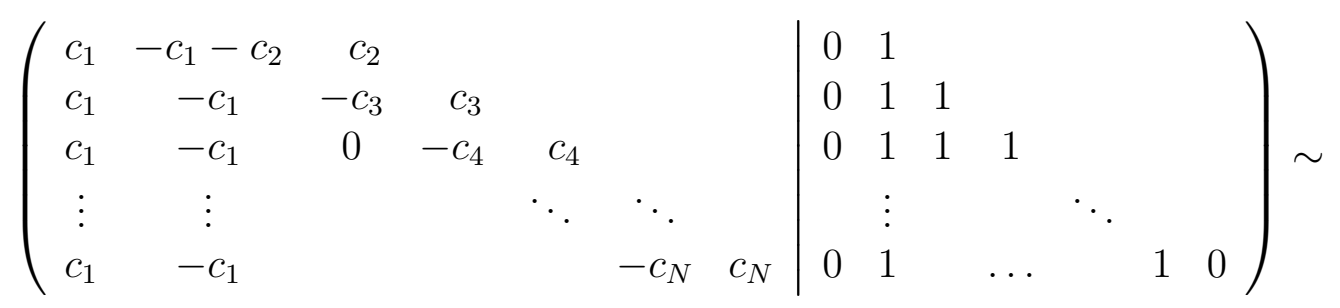

Divide by $c_{i+1}$

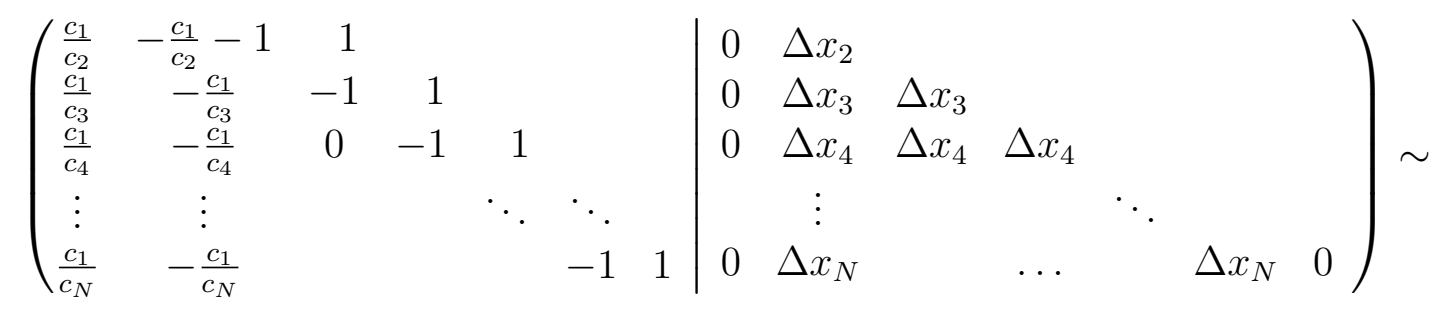

and add the rows together (first row is the sum of all rows, last row is the sum of itself).

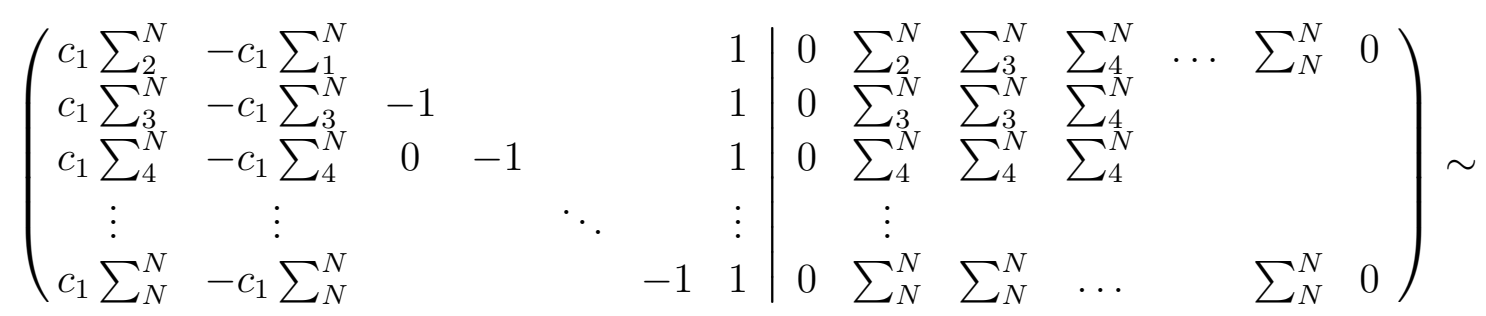

Here we have written $\sum_{i}^{N}$ instead of $\sum_{k=i}^{N} \Delta x_{k}$ in order to save space. We use the fact that $\sum_{k=1}^{N} \Delta x_{k}=1$ and that $1-\sum_{k=i+1}^{N} \Delta x_{k}=\sum_{k=1}^{i} \Delta x_{k}$. Multiply the first row by $\sum_{k=i+1}^{N} \Delta x_{k}$ and subtract it from row $i$. Thereafter multiply first row by $\Delta x_{1}$. We get

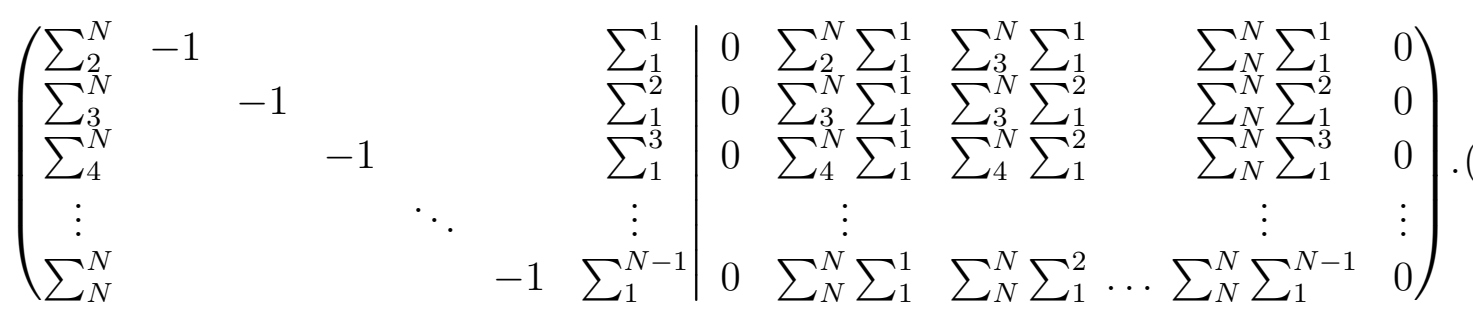


Now we need the first and last row from the whole, "original", matrix (90). Combining them with row 1 to $N-1$ in (91), we get back to the full system. Note that the mid-rows differ below and above the diagonal.

$$
\left(\begin{array}{ccc|ccc}
\tau_{0} & & & 1 & \\
\sum_{i+1}^{N} & -I & \sum_{1}^{i} & 0 & \sum_{i+1}^{N} \sum_{1}^{j} \backslash \sum_{j+1}^{N} \sum_{1}^{i} & 0 \\
& & & & & \\
& & \tau_{N} & & & 1
\end{array}\right)
$$

Multiply first and last row in (92) by $\sum_{i+1}^{N} / \tau_{0}$ and $\sum_{1}^{i} / \tau_{N}$, respectively. Subtract them from all the mid-rows. We obtain $\tilde{M}^{-1}$ as

$$
\begin{aligned}
\text { Left column }(j=0): & \left(\tilde{M}^{-1}\right)_{i 0}=\frac{1}{\tau_{0}} \sum_{k=i+1}^{N} \Delta x_{k} \\
\text { Right column }(j=N): & \left(\tilde{M}^{-1}\right)_{i N}=\frac{1}{\tau_{N}} \sum_{k=1}^{i} \Delta x_{k} \\
\text { Below diagonal }(i \geq j): & \left(\tilde{M}^{-1}\right)_{i j}=-\sum_{k=1}^{j} \Delta x_{k} \sum_{k=i+1}^{N} \Delta x_{k} \\
\text { Above diagonal }(i \leq j): & \left(\tilde{M}^{-1}\right)_{i j}=-\sum_{k=1}^{i} \Delta x_{k} \sum_{k=j+1}^{N} \Delta x_{k}
\end{aligned}
$$

\section{E The order of $G_{i j}$}

We have defined $G_{i j}=\left(\tilde{M}^{-1}\right)_{i j} F^{j}$. Assume $i \leq j$.

$$
\begin{aligned}
\left(\tilde{M}^{-1}\right)_{i j}-\left(\tilde{M}^{-1}\right)_{i j-1} & =\Delta x_{j} \sum_{k=1}^{i} \Delta x_{k} \sim \Delta x_{j} \\
\left(\tilde{M}^{-1}\right)_{i N}-\left(\tilde{M}^{-1}\right)_{i N-1} & =\left(\frac{1}{\tau_{N}}+\Delta x_{N}\right) \sum_{k=1}^{i} \Delta x_{k} \sim \Delta x_{N}
\end{aligned}
$$

where we have used that $\tau_{N} \sim 1 / \Delta x_{N}$. In the same way $\left(\tilde{M}^{-1}\right)_{i 1}-\left(\tilde{M}^{-1}\right)_{i 0} \sim \Delta x_{1}$. That is, $\left(\tilde{M}^{-1}\right)_{i j}-\left(\tilde{M}^{-1}\right)_{i j-1} \sim \Delta x_{j}$ for all $j$. The same result is also found for $i \geq j$. Together with $\left(F^{j}-F^{j-1}\right)=\Delta x_{j} F_{x}^{j}+\mathcal{O}\left(h^{2}\right)$ this yields

$$
G_{i j}-G_{i j-1}=\left(\tilde{M}^{-1}\right)_{i j} F^{j}-\left(\left(\tilde{M}^{-1}\right)_{i j}+\mathcal{O}\left(\Delta x_{j}\right)\right)\left(F^{j}+\mathcal{O}\left(\Delta x_{j}\right)\right) \sim \Delta x_{j} .
$$

Hence $G_{i j}-G_{i j-1} \sim h$.

\section{References}

[1] M. H. Carpenter, J. Nordstrom, and D. Gottlieb. A stable and conservative interface treatment of arbitrary spatial accuracy. Journal of Computational Physics, (148):341-365, 1999. 
[2] B. Diskin and J. L. Thomas. Accuracy analysis for mixed-element finite-volume discretization schemes. NIA Report 2007-08, August 2007.

[3] P. Eliasson. EDGE, a Navier-Stokes solver for unstructured grids. In Finite Volumes for Complex Applications III, pages 527-534, 2002.

[4] T. Gerhold, O. Friedrich, and J. Evans. Calculation of complex three-dimensional configurations employing the dlr-tau-code. in: AIAA paper 97-0167, 1997.

[5] B. Gustafsson, H. O. Kreiss, and J. Oliger. Time Dependent Problems and Difference Methods. Wiley-Interscience, 1995.

[6] A. Haselbacher, J. J. McGuirk, and G. J. Page. Finite volume discretization aspects for viscous flows on mixed unstructured grids. AIAA Journal, 37(2):177-184, 1999.

[7] K. Mattsson and J. Nordström. Summation by parts operators for finite difference approximations of second derivatives. Journal of Computational Physics, (199):503-540, 2004.

[8] D.J. Mavriplis and D.W. Levy. Transonic drag prediction using an unstructured multigrid solver. In Technical report, Institute for Computer Applications in Science and Engineering, 2002.

[9] E.J. Nielsen and W.K. Anderson. Recent improvements in aerodynamic design optimization on unstructured meshes. In AIAA-2001-0596, 2001.

[10] J. Nordström, K. Forsberg, C. Adamsson, and P. Eliasson. Finite volume methods, unstructured meshes and strict stability for hyperbolic problems. Applied Numerical Mathematics, 45(4):453-473, 2003.

[11] D. Schwamborn, T. Gerhold, and R. Heinrich. The DLR TAU-code: Recent applications in research and industry. In European Conference on Computational Fluid Dynamics, ECCOMAS CFD, 2006.

[12] M. Svärd, J. Gong, and J. Nordström. An accuracy evaluation of unstructured node-centered finite-volume methods. Applied Numerical Mathematics, 58:11421158, 2008.

[13] M. Svärd and J. Nordström. Stability of finite volume approximations for the laplacian operator on quadrilateral and triangular grids. Applied Numerical Mathematics, 51(1):101-125, 2004.

[14] J. L. Thomas, B. Diskin, and C. L. Rumsey. Towards verification of unstructuredgrid solvers. AIAA Journal, 46(12):3070-3079, 2008.

[15] L. Tysell and J. Nordström. Accuracy evaluation of the unstructured finite volume method in aerodynamic computations. In 10th ISGG Conference on Numerical Grid Generation, page 13, Curran Associates, Red Hook, NY 2007.

[16] J.M. Weiss, J.P. Maruszewski, and W.A. Smith. Implicit solution of preconditioned Navier-Stokes equations using algebraic multigrid. AIAA Journal, 37(1):29-36, 1999. 